\title{
Glasgow Prognostic Score is superior to ECOG PS as a prognostic factor in patients with gastric cancer with peritoneal seeding
}

\author{
SHU-QIANG YUAN ${ }^{1 *}$, RUN-CONG NIE $^{1 *}$, YONG-MING CHEN $^{1 *}$, HAI-BO QIU ${ }^{1}$, \\ XIAO-PING LI ${ }^{2}$, XIAO-JIANG CHEN ${ }^{1}$, LI-PU XU ${ }^{1}$, LI-FANG YANG ${ }^{1}$, XIAO-WEI SUN ${ }^{1}$, \\ YUAN-FANG LI ${ }^{1}$, ZHI-WEI ZHOU ${ }^{1}$, SHI CHEN $^{3 *}$ and YING-BO CHEN ${ }^{1 *}$ \\ ${ }^{1}$ Sun Yat-sen University Cancer Center, State Key Laboratory of Oncology in South China, \\ Collaborative Innovation Center for Cancer Medicine, Guangzhou, Guangdong 510060; \\ ${ }^{2}$ Department of General Surgery, Jiangmen Central Hospital, Affiliated Jiangmen Hospital of Sun Yat-sen University, \\ Jiangmen, Guangdong 529030; ${ }^{3}$ Department of Gastric Surgery, The 6th Affiliated Hospital, \\ Sun Yat-sen University, Guangzhou, Guangdong 510655, P.R. China
}

Received May 17, 2017; Accepted November 30, 2017

DOI: $10.3892 / \mathrm{ol} .2018 .7826$

\begin{abstract}
The Glasgow Prognostic Score (GPS) has been shown to be associated with survival rates in patients with advanced cancer. The present study aimed to compare the GPS with the Eastern Cooperative Oncology Group Performance Status (ECOG PS) in patients with gastric cancer with peritoneal seeding. For the investigation, a total of 384 gastric patients with peritoneal metastasis were retrospectively analyzed. Patients with elevated C-reactive protein (CRP; $>10 \mathrm{mg} / \mathrm{l})$ and hypoalbuminemia $(<35 \mathrm{mg} / \mathrm{l})$ were assigned a score of 2 . Patients were assigned a score of 1 if presenting with only one of these abnormalities, and a score of 0 if neither of these abnormalities were present. The clinicopathologic characteristics and clinical outcomes of patients with peritoneal seeding were analyzed. The results showed that the median overall survival (OS) of patients in the GPS 0 group was longer, compared with that in the GPS 1 and GPS 2 groups (15.50, vs. 10.07 and 7.97 months, respectively; $\mathrm{P}<0.001)$. No significant difference was found between the median OS of patients with
\end{abstract}

Correspondence to: Professor Ying-Bo Chen, Sun Yat-sen University Cancer Center, State Key Laboratory of Oncology in South China, Collaborative Innovation Center for Cancer Medicine, 651 East Dongfeng Road, Guangzhou, Guangdong 510060, P.R. China

E-mail: chenyb@sysucc.org.cn

Dr Shi Chen, Department of Gastric Surgery, The 6th Affiliated Hospital, Sun Yat-sen University, 26 Yuancun Erheng Road, Guangzhou, Guangdong 510655, P.R. China

E-mail: chensh47@mail.sysu.edu.cn

*Contributed equally

Key words: gastric cancer, peritoneal seeding, Glasgow Prognostic Score, performance status a good performance status $(\mathrm{ECOG}<2)$ and those with a poor (ECOG $\geq 2$ ) performance status (13.67, vs. 11.80 months; $\mathrm{P}=0.076$ ). In the subgroup analysis, the median OS in the GPS 0 group was significantly longer, compared with that in the GPS 1 and GPS 2 groups, for the patients receiving palliative chemotherapy and patients without palliative chemotherapy. Multivariate survival analysis demonstrated that CA19-9, palliative gastrectomy, first-line chemotherapy and GPS were the prognostic factors predicting OS. In conclusion, the GPS was superior to the subjective assessment of ECOG PS as a prognostic factor in predicting the outcome of gastric cancer with peritoneal seeding.

\section{Introduction}

Gastric cancer remains the second most common type of malignant cancer in China, despite the incidence decreasing worldwide $(1,2)$. In addition, the majority of patients with gastric cancer in China are diagnosed with late-stage gastric cancer (3).

Among the patterns of metastasis, peritoneal seeding is the most common and most life-threatening type of gastric cancer, and is considered to be the terminal stage of gastric cancer (4). Despite often short and poor survival rates among patients with gastric cancer with peritoneal seeding, there exists a marked heterogeneity in the survival duration. Therefore, there has been increasing interest in investigating the prognostic factors and allowing more accurate stratification for the patients, which are likely to improve clinical practice and possibly contribute to more rational study design and analysis.

The assessment of performance status as the Eastern Cooperative Oncology Group Performance Status (ECOG PS) is a simple tool to evaluate a patient's physical condition and is also a common prognostic factor predicting treatment survival rates (5). However, the ECOG PS assessment is subjective and biased. Ando et al reported that performance status assessments differed significantly among oncologists, 
nurses and patients, with the assessment by oncologists being most optimistic and that by patients the least (6). Therefore, the selection of ECOG PS as a prognostic factor remains problematic, and more objective and reliable prognostic scores are required to reflect clinical outcome in patients with advanced cancer.

There is increasing evidence that the systemic inflammatory response, as evidenced by the elevation of C-reactive protein (CRP), is critical in patients with advanced cancer $(7,8)$. Furthermore, Forrest et al reported that the Glasgow Prognostic Score (GPS), the combination of serum CRP and serum albumin, was a reliable, objective scoring tool for predicting survival rates in patients with inoperable non-small cell lung cancer (9). Additionally, several studies have demonstrated that GPS is associated with prognosis independent of age, stage and performance status in various types of malignancy (10-16).

Crumley et al reported that the GPS was superior to performance status as a prognostic factor in patients receiving palliative chemotherapy for gastroesophageal cancer (10). However, whether GPS is a superior prognostic factor to ECOG PS in predicting the survival rates of patients with gastric cancer with peritoneal seeding remains to be elucidated. Therefore, the present study aimed to compare GPS with ECOG PS in predicting the outcome of gastric cancer with peritoneal seeding.

\section{Patients and methods}

Patients. Between May 2006 and March 2014, the present study recruited 384 consecutive patients, who were diagnosed with gastric adenocarcinoma with peritoneal seeding, at Sun Yat-sen University Cancer Center. The treatment, including gastrectomy, was performed following the provision of written informed consent from patients. The present study was approved by the independent Institute Research Ethics Committee at the Sun Yat-sen University Cancer Center (Guangdong, China) and was performed according to the principles expressed in the Declaration of Helsinki.

The demographic information of the patients was collected for analysis. Only patients with an entire set of laboratory data were included in the present study. Patients who had evidence of infection, and those who received preoperative chemotherapy or radiotherapy were excluded.

The ECOG PS was evaluated by the definition of the ECOG criteria. Peritoneal seeding was classified according to the first English edition of the Japanese classification of gastric carcinoma (17). Multisite distant metastasis was defined as concurrent extra-regional lymph node metastasis, hepatic metastasis, lung metastasis or other metastases excluding peritoneal seeding. The first-line chemotherapy regimens included various agents, including 5-fluorouracil, taxane, irinotecan, oxaliplatin and capecitabine.

GPS estimation. The GPS was estimated according to a previous description (9). The patients were assigned a score of 2 if they presented with elevated CRP ( $>10 \mathrm{mg} / \mathrm{l})$ and hypoalbuminemia $(<35 \mathrm{mg} / \mathrm{l})$, a score of 1 if presenting with only one of these biochemical abnormalities, and a score of 0 if neither of these abnormalities were present.
Statistical analysis. The categorical variables are presented as numbers and percentages, and were compared using $\chi^{2}$ tests. Unadjusted Kaplan-Meier survival curves were generated to compare differences in overall survival (OS) between different groups with log-rank testing. Prognostic factors were first analyzed by univariate analysis, with which $\mathrm{P}<0.05$ was entered into multivariate analysis using Cox proportional hazard models. The forward selection method was used for multivariate Cox proportional analysis. In the present study, receiver operating characteristic (ROC) curves were also constructed to assess sensitivity, specificity and areas under the curves (AUCs) with a 95\% confidence interval (CI). $\mathrm{P}<0.05$ (two-sided) was considered to indicate a statistically significant difference. The statistical analyses described above were performed using SPSS 17.0 software (SPSS, Inc., Chicago, IL, USA).

The patients were monitored every 3 months for 2 years, and at intervals of 6-12 months thereafter until lost to follow up or mortality. The regular follow-up period ranged between 0.1 and 52.2 months (median, 9.77 months).

\section{Results}

Patient characteristics. The classified clinical and laboratory characteristics of the 384 gastric cancer patients with peritoneal seeding are shown in Table I. There were no significant differences in OS in terms of gender (male/female), ECOG PS $(<2 / \geq 2)$ (Fig. 1), tumor location (cardia/middle/antrum), signet ring cell carcinoma (yes/no), or CA72-4 $(<5.3 / \geq 5.3 \mathrm{U} / \mathrm{ml})$. By contrast, significant differences in OS were observed in terms of age, tumor size, ascites, carcinoembryonic antigen (CEA), CA19-9, albumin, CRP, peritoneal seeding classification, multisite distant metastasis, palliative gastrectomy, first-line chemotherapy and GPS (Fig. 2).

The associations between clinicopathological characteristics and GPS in patients with gastric cancer with peritoneal seeding are shown in Table II. Age, gender, ascites, CA72-4, albumin, CRP, classification of peritoneal seeding, multisite distant metastasis and palliative gastrectomy were closely associated with the GPS classification. Compared with the GPS 0 and GPS 1 patients, the GPS 2 patients appeared to have higher levels of tumor marker and CRP, and had a higher frequency of ascites and multisite distant metastasis with more severe peritoneal seeding.

Survival rates. The results of the Kaplan-Meier analysis demonstrated that patients with good performance status $(\mathrm{ECOG}<2)$ had longer median OS, compared with those with poor performance status $(\mathrm{ECOG} \geq 2)$, with an $\mathrm{OS}$ of 13.67 (95\% CI: 11.39-15.94), vs. 11.80 (95\% CI: 9.40-14.20) months, respectively. However, this difference was not significant $(\mathrm{P}=0.076$; Fig. 1 and Table I).

However, the Kaplan-Meier analysis demonstrated that patients in the GPS 0 group had a significantly longer median OS, compared with those in the GPS 1 and GPS 2 group, with median OS rates of 15.50 (95\% CI: 13.09-17.91), 10.07 (95\% CI: 8.29-11.84) and 7.97 (95\% CI: 6.47-9.46) months, respectively $(\mathrm{P}<0.001$; Fig. 2 and Table I). The ROC curves also showed that the AUC of GPS was $0.613(\mathrm{P}=0.011)$, whereas the AUC of ECOG PS was 0.552 ( $\mathrm{P}=0.243$; Fig. 3). 
Table I. Classified clinical and laboratory characteristics associated with OS.

\begin{tabular}{|c|c|c|c|c|c|c|c|}
\hline & & & & & & & \\
\hline & Dotiontc (n) & OS (months), & D & Characteristic & Patients (n) & $\begin{array}{l}\text { OS (months), } \\
\text { median }(95 \% \mathrm{CI})\end{array}$ & P-value \\
\hline & & & & First-line & & & $<0.001$ \\
\hline Age (years) & & & 0.019 & chemotherapy & & & \\
\hline$<65$ & 319 & $14.03(12.07-16.00)$ & & Yes & 279 & $15.40(13.70-17.10)$ & \\
\hline$\geq 65$ & 65 & $10.37(6.78-13.96)$ & & No & 105 & $6.40(4.66-8.14)$ & \\
\hline Gender (n) & & & 0.285 & GPS & & & $<0.001$ \\
\hline Male & 210 & $12.23(9.94-14.53)$ & & 0 & 247 & $15.50(13.09-17.91)$ & \\
\hline Female & 174 & $13.70(11.99-15.41)$ & & 1 & 101 & $10.07(8.29-11.84)$ & \\
\hline ECOG PS (n) & & & 0.076 & 2 & 36 & $7.97(6.47-9.46)$ & \\
\hline
\end{tabular}

$<2$

$\geq 2$

99

11.80 (9.40-14.20)

Tumor location

$\begin{array}{lrl}\text { Cardia } & 96 & 11.73(8.78-14.69) \\ \text { Middle } & 142 & 13.13(10.07-16.20) \\ \text { Antrum } & 138 & 14.23(11.37-17.10)\end{array}$

Tumor size $(\mathrm{cm})$

$<5$

$\geq 5$

SRCC

Yes

No

150

$14.27(10.83-17.71)$

202

$11.27(8.72-13.81)$

$136 \quad 15.83(12.19-19.47)$

$244 \quad 11.77$ (9.51-14.02)

Ascites

Yes

No

160

$9.73(8.21-11.25)$

CEA (ng/ml)

$<5$
$>5$

$\geq 5$

CA19-9 (U/ml)

$<35$

$\geq 35$

CA72-4 (U/ml)

$<5.3$

$\geq 5.3$

Albumin (g/l)

$<35$

$\geq 35$

CRP (mg/l)

$<10$

$\geq 10$

Peritoneal seeding

$\begin{array}{lll}\mathrm{P} 1 / \mathrm{P} 2 & 200 & 15.47(13.73-17.20) \\ \mathrm{P} 3 & 184 & 10.00(8.64-11.36)\end{array}$

Multisite distant

metastasis

$$
\text { Yes }
$$

142

$10.17(7.75-12.59)$

No

15.47 (12.66-18.27)

Palliative gastrectomy

Yes

164

No

219

Table I. Continued.

GPS, Glasgow Prognostic Score; ECOG PS, Eastern Cooperative Oncology Group Performance Status; SRCC, signet ring cell carcinoma; CEA, baseline carcinoembryonic antigen; CA19-9, baseline carbohydrate antigen 19-9; CA72-4 baseline carbohydrate antigen 72-4; CRP, C-reactive protein.

0.038

0.052

0.001

$15.73(13.01-18.46)$

$14.50(12.90-16.10)$

0.005

$9.80(7.27-12.33)$

$15.50(12.76-18.25)$

$<0.001$

$10.90(7.95-13.86)$

0.125

$14.00(11.55-16.46)$

$12.10(8.82-15.38)$

0.001

8.97 (7.51-10.42)

14.03 (12.34-15.73)

14.57 (12.80-16.34)

$<0.001$

$8.73(7.30-10.17)$

0.001

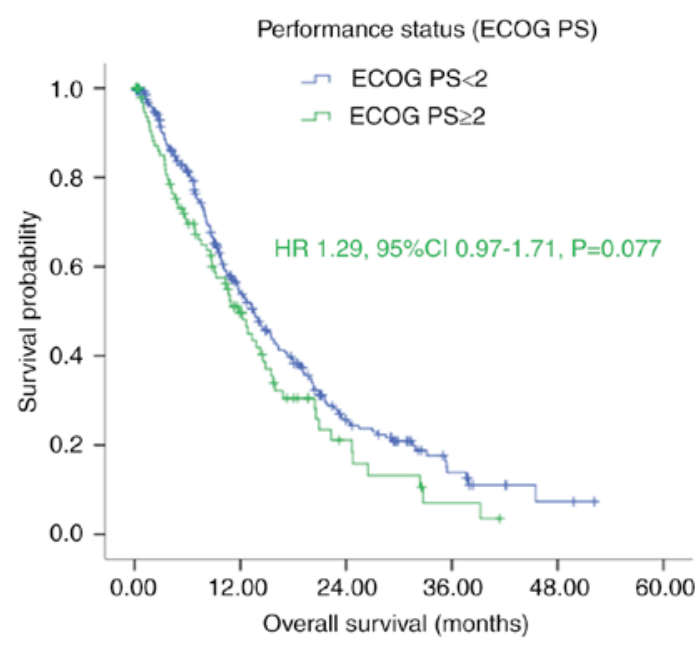

Figure 1. Kaplan-Meier survival curves of patients with gastric cancer with peritoneal seeding according to ECOG PS $(\mathrm{P}=0.077)$. P-values were calculated using the log-rank test. ECOG PS, Eastern Cooperative Oncology Group Performance Status.

In the subgroup analysis, with first-line chemotherapy, the median OS of patients in the GPS 0 group was significantly longer, compared with the median OS of patients in the GPS 1 and GPS 2 group [17.40 (95\% CI: 14.47-20.33), vs. 11.67 (95\% CI: 8.50-14.84), vs. 8.97 (95\% CI: 7.20-10.73) months, respectively], as shown in Fig. $4 \mathrm{~A}(\mathrm{P}=0.008)$. Without first-line chemotherapy, patients in the GPS 0 group also had a significantly longer median OS, compared with those in the GPS 1 and GPS 2 groups [10.00 (95\% CI: 5.05-14.95), vs. 3.73 (95\% CI: 0.00-7.58), vs. 3.63 (95\% CI: 2.30-4.97) months, respectively], as shown in Fig. $4 \mathrm{~B}(\mathrm{P}=0.005)$.

In patients with multisite distant metastasis, the median OS of the GPS 0 group was longer, compared with the median OS in the GPS 1 and GPS 2 group [12.43 (95\% CI: 9.75-15.12), vs. 9.20 (95\% CI: 5.75-12.65), vs. 4.73 (95\% CI: 3.07-6.40) months (Fig. 5A; $\mathrm{P}=0.002$ ). Without multisite distant metastasis, the GPS 0 group also had a longer median OS, compared 
Table II. Clinicopathlogical characteristics of 384 patients with gastric cancer with peritoneal metastasis according to GPS.

\begin{tabular}{lrrr}
\hline Characteristic & GPS 0 (\%) & GPS 1 (\%) GPS 2 $(\%)$ \\
\hline Patients (n) & $247(64.3)$ & $101(26.3)$ & $36(9.4)$ \\
Age (years) & & & \\
$<65$ & $215(87.0)$ & $77(76.2)$ & $27(75.0)$ \\
$\geq 65$ & $32(13.0)$ & $24(23.8)$ & $9(25.0)$
\end{tabular}

Gender (n)

Male $\quad 121(49.0) \quad 65(64.4) \quad 24(66.7)$

Female $\quad 126(51.0) \quad 36(35.6) \quad 12(33.3)$

ECOG PS (n)

$\begin{array}{lrrr}<2 & 191(77.3) & 71(70.3) & 23(63.9) \\ \geq 2 & 56(22.7) & 30(29.7) & 13(36.1)\end{array}$

Tumor location

$\begin{array}{lllr}\text { Cardia } & 66(27.4) & 23(23.2) & 7(19.4) \\ \text { Middle } & 89(36.9) & 36(36.4) & 17(47.2) \\ \text { Antrum } & 86(35.7) & 40(40.4) & 12(33.3)\end{array}$

Tumor

size $(\mathrm{cm})$

$\begin{array}{lllr}<5 & 105(46.3) & 36(38.7) & 9(28.1) \\ \geq 5 & 122(53.7) & 57(61.3) & 23(71.9)\end{array}$

SRCC

Yes

No

$156(63.4) \quad 61(61.6) \quad 27(77.1)$

Ascites

Yes

No

$90(36.6) \quad 38(38.4) \quad 8(22.9)$

CEA (ng/ml)

$<5$

$\geq 5$

67 (67.6) $\quad 47(46.5) \quad 10(27.8)$

$80(32.4) \quad 54(53.5) \quad 26(72.2)$

$181(75.7) \quad 70(70.0) \quad 19(59.4)$

$58(24.3) \quad 30(30.0) \quad 13(40.6)$

CA19-9 (U/ml)

$<35 \quad 147(62.8) \quad 58(58.6) \quad 18(58.1)$

$\begin{array}{llll}\geq 35 & 87(37.2) & 41(41.4) & 13(41.9)\end{array}$

CA72-4 (U/ml)

$<5.3$

$\geq 5.3$

$116(56.9) \quad 37(45.1) \quad 8(27.6)$

Albumin (g/l)

$<35$

$\geq 35$

CRP (mg/l)

$<10$

$\geq 10$

$88(43.1) \quad 45(54.9) \quad 21(72.4)$

Peritoneal

seeding

$\begin{array}{llll}\mathrm{P} 1 / \mathrm{P} 2 & 145(58.7) & 44(43.6) & 11(30.6) \\ \mathrm{P} 3 & 102(41.3) & 57(56.4) & 25(69.4)\end{array}$

Multisite distant

metastasis

$\begin{array}{lrrr}\text { Yes } & 75(30.5) & 55(54.5) & 12(33.3) \\ \text { No } & 171(69.5) & 46(45.5) & 24(66.7)\end{array}$

Palliative

gastrectomy

$\begin{array}{llll}\text { Yes } & 122(49.6) & 32(31.7) & 10(27.8) \\ \text { No } & 124(50.4) & 69(68.3) & 26(72.2)\end{array}$

0.010

0.636

0.102

0.715

0.006

$<0.001$

$0(0.0) \quad 27(26.7) \quad 36(100.0)$

$247(100.0) \quad 74(73.3) \quad 0(0.0)$

$247(100.0) \quad 74(73.3) \quad 0(0.0)$

$0(0.0) \quad 27(26.7) \quad 36(100.0)$

$<0.001$

0.001

$<0.001$

0.001
Table II. Continued.

Characteristic GPS $0(\%)$ GPS $1(\%)$ GPS $2(\%)$ P-value

First-line

0.242

chemotherapy

$\begin{array}{lrrr}\text { Yes } & 186(75.3) & 70(69.3) & 23(63.9) \\ \text { No } & 61(24.7) & 31(30.7) & 13(36.1)\end{array}$

GPS, Glasgow Prognostic Score; ECOG PS, Eastern Cooperative Oncology Group Performance Status; SRCC, signet ring cell carcinoma; CEA, baseline carcinoembryonic antigen; CA19-9, baseline carbohydrate antigen 19-9; CA72-4 baseline carbohydrate antigen 72-4; CRP, C-reactive protein.

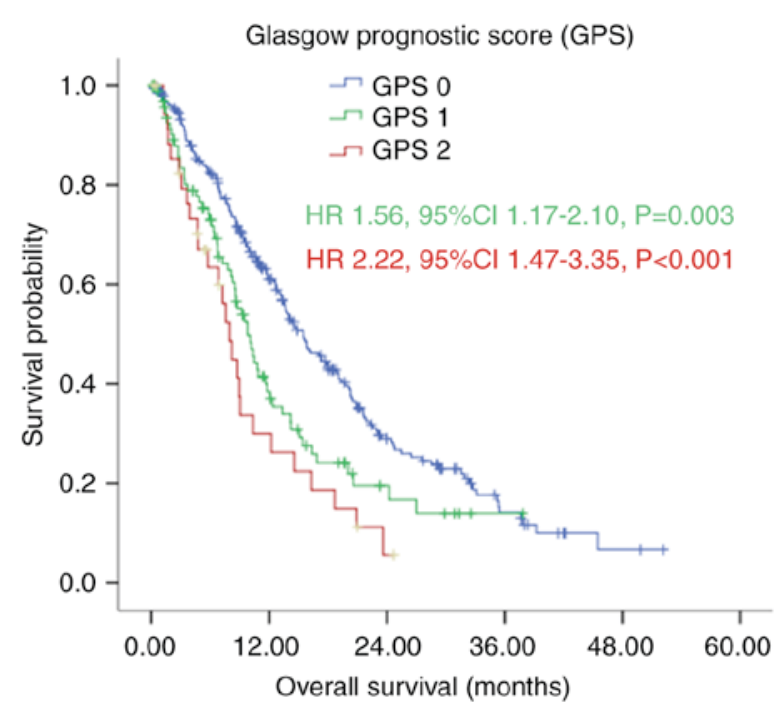

Figure 2. Kaplan-Meier survival curves of patients with gastric cancer with peritoneal seeding according to GPS $(\mathrm{P}<0.001)$. P-values were calculated using the log-rank test. GPS, Glasgow Prognostic Score.

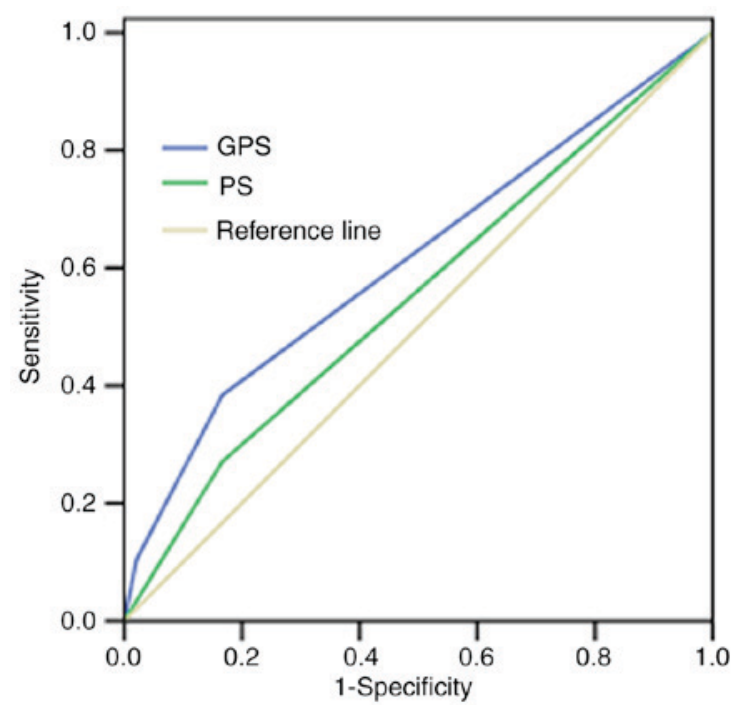

Figure 3. Receiver operating characteristics curves of GPS and ECOG PS of patients with gastric cancer with peritoneal seeding. The AUC of GPS was $0.613(\mathrm{P}=0.011)$, the AUC of ECOG PS was $0.552(\mathrm{P}=0.243)$. GPS, Glasgow Prognostic Score; ECOG PS, Eastern Cooperative Oncology Group Performance Status; AUC, area under the curve. 
Table III. Univariate and multivariate of analyses of overall survival in patients with gastric cancer with peritoneal metastasis.

\begin{tabular}{|c|c|c|c|c|}
\hline \multirow[b]{2}{*}{ Variable } & \multicolumn{2}{|c|}{ Univariate analysis } & \multicolumn{2}{|c|}{ Multivariate analysis } \\
\hline & HR (95\%CI) & $\mathrm{P}$-value & HR (95\%CI) & P-value \\
\hline Age (years) & & 0.020 & & \\
\hline$<65$ & 1 & & & \\
\hline$\geq 65$ & $1.44(1.60-1.96)$ & & & \\
\hline Gender (n) & & 0.285 & & \\
\hline Male & 1 & & & \\
\hline Female & $0.87(0.68-1.12)$ & & & \\
\hline ECOG PS (n) & & 0.077 & & \\
\hline$<2$ & 1 & & & \\
\hline$\geq 2$ & $1.29(0.97-1.71)$ & & & \\
\hline Tumor location & & 0.515 & & \\
\hline Cardia & 1 & & & \\
\hline Middle & 1.07 (0.77-1.47) & 0.696 & & \\
\hline Antrum & $0.90(0.65-1.25)$ & 0.536 & & \\
\hline Tumor size $(\mathrm{cm})$ & & 0.039 & & \\
\hline$<5$ & 1 & & & \\
\hline$\geq 5$ & $1.32(1.01-1.71)$ & & & \\
\hline SRCC & & 0.053 & & \\
\hline No & 1 & & & \\
\hline Yes & $0.77(0.59-1.00)$ & & & \\
\hline Ascites & & $<0.001$ & & \\
\hline No & 1 & & & \\
\hline Yes & $1.53(1.19-1.97)$ & & & \\
\hline CEA (ng/ml) & & 0.006 & & \\
\hline$<5$ & 1 & & & \\
\hline$\geq 5$ & $1.47(1.12-1.93)$ & & & \\
\hline CA19-9 (U/ml) & & $<0.001$ & & $<0.001$ \\
\hline$<35$ & 1 & & 1 & \\
\hline$\geq 35$ & $1.62(1.25-2.10)$ & & $1.63(1.25-2.12)$ & \\
\hline CA72-4 (U/ml) & & 0.126 & & \\
\hline$<5.3$ & 1 & & & \\
\hline$\geq 5.3$ & $1.24(0.94-1.62)$ & & & \\
\hline Albumin (g/l) & & 0.001 & & \\
\hline$<35$ & 1 & & & \\
\hline$\geq 35$ & $0.59(0.43-0.82)$ & & & \\
\hline CRP (mg/l) & & $<0.001$ & & \\
\hline$<10$ & 1 & & & \\
\hline$\geq 10$ & $1.72(1.30-2.27)$ & & & \\
\hline Peritoneal seeding & & 0.001 & & \\
\hline $\mathrm{P} 1 / \mathrm{P} 2$ & 1 & & & \\
\hline P3 & $1.50(1.17-1.92)$ & & & \\
\hline Multisite distant metastasis & & 0.002 & & \\
\hline No & 1 & & & \\
\hline Yes & $1.49(1.15-1.93)$ & & & \\
\hline Palliative gastrectomy & & $<0.001$ & & $<0.001$ \\
\hline No & 1 & & 1 & \\
\hline Yes & $0.51(0.40-0.66)$ & & $0.56(0.43-0.73)$ & \\
\hline First-line chemotherapy & & $<0.001$ & & $<0.001$ \\
\hline No & 1 & & 1 & \\
\hline Yes & $0.43(0.32-0.57)$ & & $0.40(0.30-0.54)$ & \\
\hline
\end{tabular}


Table III. Continued.

\begin{tabular}{|c|c|c|c|c|}
\hline \multirow[b]{2}{*}{ Variable } & \multicolumn{2}{|c|}{ Univariate analysis } & \multicolumn{2}{|c|}{ Multivariate analysis } \\
\hline & $\mathrm{HR}(95 \% \mathrm{CI})$ & P-value & $\mathrm{HR}(95 \% \mathrm{CI})$ & P-value \\
\hline GPS & & $<0.001$ & & 0.006 \\
\hline 0 & 1 & & 1 & \\
\hline 1 & $1.56(1.17-2.10)$ & 0.003 & $1.47(1.08-1.98)$ & 0.013 \\
\hline 2 & $2.22(1.47-3.35)$ & $<0.001$ & $1.76(1.13-2.73)$ & 0.012 \\
\hline
\end{tabular}

GPS, Glasgow Prognostic Score; ECOG PS, Eastern Cooperative Oncology Group Performance Status; SRCC, signet ring cell carcinoma; CEA, baseline carcinoembryonic antigen; CA19-9, baseline carbohydrate antigen 19-9; CA72-4 baseline carbohydrate antigen 72-4; CRP, C-reactive protein.

A



B

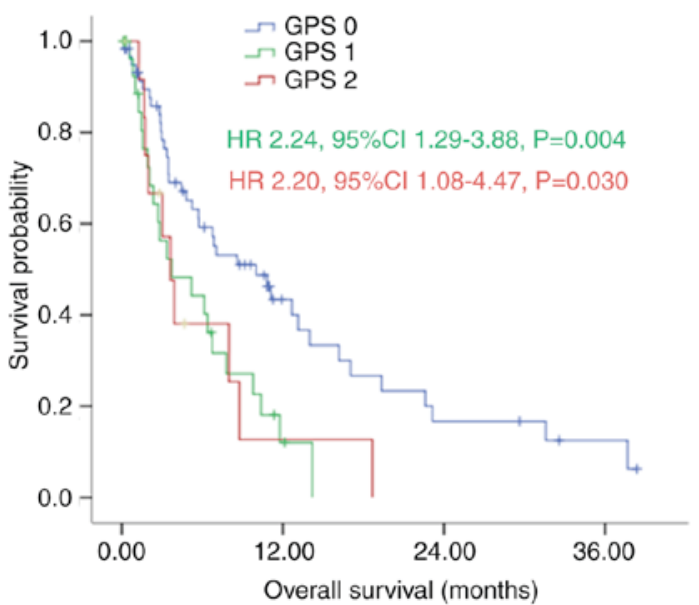

Figure 4. Kaplan-Meier survival curves of patients with gastric cancer with peritoneal seeding according to GPS stratified by first-line chemotherapy. (A) With first-line chemotherapy ( $\mathrm{P}=0.008)$; $(\mathrm{B})$ without first-line chemotherapy $(\mathrm{P}=0.005)$. P-values were calculated using the log-rank test. GPS, Glasgow Prognostic Score.

with the median OS in the GPS 1 and GPS 2 groups [17.40 (95\% CI: 14.12-20.68), vs. 10.37 \{95\% CI: 7.97-12.76), vs. 9.03 (95\% CI: 6.12-11.95) months, as shown in Fig. 5B ( $\mathrm{P}=0.019)$.

Univariate and multivariate analyses. In the univariate survival analysis, age $(\mathrm{P}=0.020)$, tumor size $(\mathrm{P}=0.039)$, ascites $(\mathrm{P}<0.001)$, CEA $(\mathrm{P}=0.006)$, CA19-9 $(\mathrm{P}<0.001)$, albumin $(\mathrm{P}=0.001), \mathrm{CRP}(\mathrm{P}<0.001)$, classification of peritoneal seeding $(\mathrm{P}=0.001)$, multisite distant metastasis $(\mathrm{P}=0.002)$, palliative gastrectomy $(\mathrm{P}<0.001)$, first-line chemotherapy $(\mathrm{P}<0.001)$ and GPS $(\mathrm{P}<0.001)$ were associated with OS (Table III). The multivariate survival analysis demonstrated that CA19-9 $(\mathrm{P}<0.001)$, palliative gastrectomy $(\mathrm{P}<0.001)$, first-line chemotherapy $(\mathrm{P}<0.001)$ and GPS $(\mathrm{P}<0.001)$ remained the prognostic factors in predicting the OS (Table III).

\section{Discussion}

There is substantial evidence that tumor-related factors and host-related factors, including poor performance status, weight loss and systemic inflammatory response, can determine the outcomes of patients with malignant cancer $(15,18)$. However, the assessments of weight loss and performance status are subjective and biased. By contrast, in the present study, univariate and multivariate analysis demonstrated that an inflammatory prognostic score, as evidenced by the GPS, was superior to performance status (ECOG PS) as a prognostic factor in predicting the outcome of patients with gastric cancer with peritoneal dissemination.

It is generally recognized that cancer-related inflammation can assist in malignant cancer cell proliferation and survival, accelerating angiogenesis and metastasis, destroying the adaptive immune responses of the patients, and finally altering the responses of patients to hormones and chemotherapy treatment (19). CRP is an important acute phase protein and a sensitive marker of the systemic inflammatory response. Additionally, CRP can be expressed in malignant cancer cells $(7,20,21)$. CRP synthesis is generally induced by several chemokines and cytokines, including tumor necrosis factor- $\alpha$ (TNF- $\alpha$ ), interleukin-1 (IL-1) and IL-6, from the liver or cancer tissues $(22,23)$. However, serum CRP measurement is more convenient and stable, compared with cytokine and chemokine measurement. Several studies have revealed that elevated CRP is associated with poor survival rates in certain 
Table IV. Response to chemotherapy of 278 patients with gastric cancer with peritoneal metastasis according to GPS.

\begin{tabular}{lcccr}
\hline Characteristic & GPS 0 & GPS 1 & GPS 2 & P-value \\
\hline Patients (n) & 185 & 70 & 23 & \\
Period of chemotherapy, mean (n, 95\% CI) & $5.39(4.82-5.97)$ & $5.06(4.14-5.97)$ & $4.96(3.58-6.33)$ & 0.762 \\
DCR (CR+PR+SD) n (\%) & $48(25.9)$ & $19(27.1)$ & $3(13)$ & 0.368 \\
\hline
\end{tabular}

GPS, Glasgow Prognostic Score; DCR, disease control rate; CR, complete response; PR, partial response; SD, stable disease; CI, confidence interval.
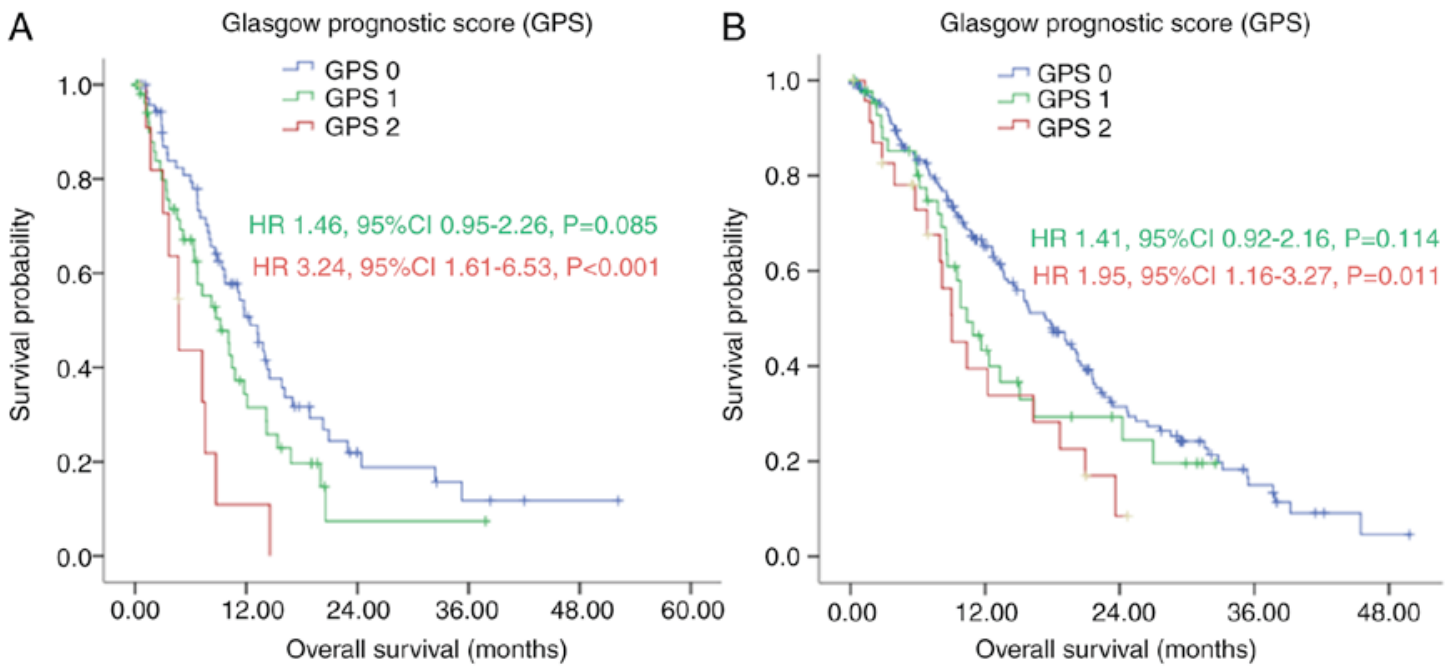

Figure 5. Kaplan-Meier survival curves of patients with gastric cancer with peritoneal seeding according to GPS stratified by multisite distant metastasis (A) With multisite distant metastasis ( $\mathrm{P}=0.002)$; (B) without multisite distant metastasis $(\mathrm{P}=0.019)$. $\mathrm{P}$-values were calculated using the log-rank test. GPS, Glasgow Prognostic Score.

types of malignant cancer, including breast cancer, hepatocellular carcinoma and gastric cancer $(8,24,25)$. In accordance with other studies $(8,24)$, the present study showed that elevated CRP correlated with poorer prognosis in patients with gastric cancer and peritoneal seeding.

Lien et al reported that preoperative serum albumin levels are associated with resectability and survival rates in patients with gastric cancer (26). Serum albumin is not only an indicator used to recognize the nutritional status of patients, but is also useful for predicting the prognostic outcome of cancer patients $(5,27)$. Patients with gastric cancer with peritoneal seeding often develop hypoalbuminemia due to oral intake deficiency, overconsumption, bleeding and ascites. In the present study, univariate analysis revealed that hypoalbuminemia was significantly associated with poor prognosis. However, when CRP and albumin were placed in the multivariate analysis, neither of them was associated with OS, which indicated the insufficiency of serum CRP and albumin alone as a prognostication. Therefore, GPS, the combination of serum albumin and CRP, was superior in predicting the outcome of gastric cancer with peritoneal seeding.

The mechanism by which GPS affect cancer survival rates remains to be fully elucidated. However, in addition to reflecting the presence of a systemic inflammatory response, GPS may also reflect the declining nutrition status of patients with advanced stage disease, which affects their tolerance and compliance to therapeutic regimens (10). In the present study, it was also noted that a higher GPS correlated significantly with higher levels of tumor markers, increased frequency of ascites, multisite distant metastasis and more severe peritoneal seeding, suggesting that a higher GPS was correlated with a more aggressive disease phenotype. However, certain therapeutic regimes can lead to elevated CRP or weight loss and malnourishment, and thus reduced albumin levels. Therefore, whether the poorer survival rate of patients was due to these host-associated factors or cancer-associated factors also remains to be elucidated. Of note, the present study found that there were no significant differences among the GPS 0, GPS 1 and GPS 2 groups regarding the period of first-line chemotherapy and response to chemotherapy (Table IV), which was a contradiction to the findings of other studies $(12,20)$. This suggested that patients with a higher GPS should also receive active palliative chemotherapy. Although anti-inflammatory treatment with low-dose aspirin lowers the incidence of colorectal adenomas and the mortality rate of several common types of cancer $(28,29)$, whether anti-inflammatory treatment can improve the outcome of patients with advanced cancer remains to be elucidated. Furthermore, whether a higher GPS is a cause or a consequence of cancer progression also remains unclear.

Irrespective of the mechanisms involved, the results of the present study showed that GPS was a simple, objective and reliable survival predictor for gastric cancer with 
peritoneal seeding, which was true for those receiving palliative chemotherapy and for those who were not.

The present study was a substantially retrospective study, which is a potential limitation. Although the data in the present study were from a high-volume institution, the results require cautious interpretation and a large-scale prospective study is required to validate the results.

In conclusion, the present study indicated that the GPS is superior to performance status (ECOG PS) as a prognostic factor in predicting the outcome of patients with gastric cancer with peritoneal seeding.

\section{Acknowledgements}

This study was supported in part by a grant from National Natural Science Foundation of China (grant no. 81302144) and the Guangdong Science and Technology Department (grant no. 2012B0617000879).

\section{References}

1. Chen W, Zheng R, Baade PD, Zhang S, Zeng H, Bray F, Jemal A Yu XQ and He J: Cancer statistics in China, 2015. CA Cancer J Clin 66: 115-132, 2016.

2. Torre LA, Bray F, Siegel RL, Ferlay J, Lortet-Tieulent J and Jemal A: Global cancer statistics, 2012. CA Cancer J Clin 65 : 87-108, 2015.

3. Chen S, Li YF, Feng XY, Zhou ZW, Yuan XH and Chen YB: Significance of palliative gastrectomy for late-stage gastric cancer patients. J Surg Oncol 106: 862-871, 2012.

4. Bando E, Yonemura Y, Takeshita Y, Taniguchi K, Yasui T, Yoshimitsu Y, Fushida S, Fujimura T, Nishimura G and Miwa K: Intraoperative lavage for cytological examination in 1,297 patients with gastric carcinoma. Am J Surg 178: 256-262, 1999.

5. Sachlova M, Majek O and Tucek S: Prognostic value of scores based on malnutrition or systemic inflammatory response in patients with metastatic or recurrent gastric cancer. Nutr Cancer 66: 1362-1370, 2014.

6. Ando M, Ando Y, Hasegawa Y, Shimokata K, Minami H, Wakai K, Ohno Y and Sakai S: Prognostic value of performance status assessed by patients themselves, nurses, and oncologists in advanced non-small cell lung cancer. Br J Cancer 85: 1634-1639, 2001.

7. Nozoe T, Korenaga D, Futatsugi M, Saeki H, Maehara Y and Sugimachi K: Immunohistochemical expression of C-reactive protein in squamous cell carcinoma of the esophagus-significance as a tumor marker. Cancer Lett 192: 89-95, 2003.

8. Hashimoto K, Ikeda Y, Korenaga D, Tanoue K, Hamatake M, Kawasaki K, Yamaoka T, Iwatani Y, Akazawa K and Takenaka K: The impact of preoperative serum C-reactive protein on the prognosis of patients with hepatocellular carcinoma. Cancer 103: $1856-1864,2005$.

9. Forrest LM, McMillan DC, McArdle CS, Angerson WJ and Dunlop DJ: Evaluation of cumulative prognostic scores based on the systemic inflammatory response in patients with inoperable non-small-cell lung cancer. Br J Cancer 89: 1028-1030, 2003.

10. Crumley AB, McMillan DC, McKernan M, McDonald AC and Stuart RC: Evaluation of an inflammation-based prognostic score in patients with inoperable gastro-oesophageal cancer. Br J Cancer 94: 637-641, 2006.

11. Ishizuka M, Nagata H, Takagi K, Horie $T$ and Kubota K: Inflammation-based prognostic score is a novel predictor of postoperative outcome in patients with colorectal cancer. Ann Surg 246: 1047-1051, 2007.
12. Crumley AB, Stuart RC, McKernan M, McDonald AC and McMillan DC: Comparison of an inflammation-based prognostic score (GPS) with performance status (ECOG-ps) in patients receiving palliative chemotherapy for gastroesophageal cancer. J Gastroenterol Hepatol 23: e325-e329, 2008.

13. Roxburgh CS, Crozier JE, Maxwell F, Foulis AK, Brown J, McKee RF, Anderson JH, Horgan PG and McMillan DC: Comparison of tumour-based (Petersen Index) and inflammation-based (Glasgow Prognostic Score) scoring systems in patients undergoing curative resection for colon cancer. Br J Cancer 100: 701-706, 2009.

14. Kinoshita A, Onoda H, Imai N, Iwaku A, Oishi M, Fushiya N, Koike K, Nishino H and Tajiri H: Comparison of the prognostic value of inflammation-based prognostic scores in patients with hepatocellular carcinoma. Br J Cancer 107: 988-993, 2012.

15. Roxburgh CS and McMillan DC: Role of systemic inflammatory response in predicting survival in patients with primary operable cancer. Future Oncol 6: 149-163, 2010

16. Gao Y and Huang D: The value of the systematic inflammation-based Glasgow Prognostic Score in patients with gastric cancer: A literature review. J Cancer Res Ther 10: 799-804, 2014.

17. Japanese Research Society for Gastric Cancer. Japanese Classification of Gastric Carcinoma. 1st English edition. Tokyo, Kanehara \& Co, Ltd, 1995.

18. Andreyev HJ, Norman AR, Oates J and Cunningham D: Why do patients with weight loss have a worse outcome when undergoing chemotherapy for gastrointestinal malignancies? Eur J Cancer 34: 503-509, 1998.

19. Mantovani A, Allavena P, Sica A and Balkwill F: Cancer-related inflammation. Nature 454: 436-444, 2008.

20. Hwang JE, Kim HN, Kim DE, Choi HJ, Jung SH, Shim HJ, Bae WK, Hwang EC, Cho SH and Chung IJ: Prognostic significance of a systemic inflammatory response in patients receiving first-line palliative chemotherapy for recurred or metastatic gastric cancer. BMC Cancer 11: 489, 2011.

21. Du Clos TW: Function of C-reactive protein. Ann Med 32: 274-278, 2000

22. Du Clos TW and Mold C: C-reactive protein: An activator of innate immunity and a modulator of adaptive immunity. Immunol Res 30: 261-277, 2004

23. Slaviero KA, Clarke SJ and Rivory LP: Inflammatory response: An unrecognised source of variability in the pharmacokinetics and pharmacodynamics of cancer chemotherapy. Lancet Oncol 4: 224-232, 2003

24. McMillan DC, Elahi MM, Sattar N, Angerson WJ, Johnstone J and McArdle CS: Measurement of the systemic inflammatory response predicts cancer-specific and non-cancer survival in patients with cancer. Nutr Cancer 41: 64-69, 2001.

25. Imaoka H, Mizuno N, Hara K, Hijioka S, Tajika M, Tanaka T, Ishihara M, Yogi T, Tsutsumi H, Fujiyoshi T, et al: Evaluation of modified glasgow prognostic score for pancreatic cancer: A retrospective cohort study. Pancreas 45: 211-217, 2016.

26. Lien YC, Hsieh CC, Wu YC, Hsu HS, Hsu WH, Wang LS, Huang MH and Huang BS: Preoperative serum albumin level is a prognostic indicator for adenocarcinoma of the gastric cardia. J Gastrointest Surg 8: 1041-1048, 2004.

27. Lee J, Lim T, Uhm JE, Park KW, Park SH, Lee SC, Park JO, Park YS, Lim HY, Sohn TS, et al: Prognostic model to predict survival following first-line chemotherapy in patients with metastatic gastric adenocarcinoma. Ann Oncol 18: 886-891, 2007.

28. Baron JA, Cole BF, Sandler RS, Haile RW, Ahnen D, Bresalier R, McKeown-Eyssen G, Summers RW, Rothstein R, Burke CA, et al: A randomized trial of aspirin to prevent colorectal adenomas. N Engl J Med 348: 891-899, 2003.

29. Rothwell PM, Fowkes FG, Belch JF, Ogawa H, Warlow CP and Meade TW: Effect of daily aspirin on long-term risk of death due to cancer: Analysis of individual patient data from randomised trials. Lancet 377: 31-41, 2011. 